\title{
Application of ethylene glycol in ventilation systems with variable air flow
}

\author{
Vitaly Shichkin ${ }^{1}$, Maria Zherlykina ${ }^{1, *}$, Roman Sheps ${ }^{1}$, Sergey Yaremenko ${ }^{1}$ \\ ${ }^{1}$ Voronezh State Technical University, Moscovskiy prospect, 14, Voronezh, 394026, Russia
}

\begin{abstract}
The relevance of the use of ethylene glycol as an intermediate heat carrier in the ventilation system during the treatment of supply air is justified. A description of the operation scheme of a cooling system with an intermediate heat carrier at a variable supply air flow rate is presented. On the Id-diagram of the state of the humid air, the construction of air treatment processes in the central air conditioner for all purposes of the transformed room is performed. The authors have established the need for cooling and dehumidification of the supply air in all operating modes. In order to determine the maximum possible load on the elements of the building's air conditioning system, the type of room with the highest supply air consumption was selected and the air treatment process was constructed on an Id diagram at different outdoor temperatures. A method for calculating the parameters of the cooling medium is developed and graphs are constructed that allow us to establish the relationship between the parameters of the heat exchange equipment and the purpose of the room. The regularities in the change of the parameters of the heat and cold carrier when regulating the operation of the system depending on the change in the load on it are revealed. The operating ranges of the proposed scheme of air treatment with an intermediate heat carrier in the warm period of the year with variable flow rate of the treated air and various parameters of the outdoor air are given. Based on this, the possibility of creating an algorithm for automating the ventilation system adapted for buildings of cultural and entertainment purposes is justified.
\end{abstract}

\section{Introduction}

At present, in the modern urban planning of the Russian Federation, a promising and at the same time complex format is widely used - multifunctional centers or complexes. These include buildings for both residential and public use, sports and concert venues. Also, facilities are being built in which the multidisciplinary functional load is concentrated in a single large-volume room. The transformable room is a unique format of the venue for events, in which in a few minutes a single spacious hall can be divided into several fully functional modules for efficient use of the working space.

It should be noted that the multi-level and diverse mode of operation of the room must be considered when creating and automatically maintaining the parameters of the internal

\footnotetext{
* Corresponding author: Kafedravgasu@yandex.ru
} 
air that are comfortable for people to stay in it. The task of the air conditioning system of the transformed room is to ensure the normalized parameters of the internal air, depending on its purpose and volume, which may be different at different intervals of time.

At the same time, assessing the type and amount of harmful substances entering the room, it was found that the dominant factor is the heat from people, which, in turn, is a variable value depending on the purpose of the transformed room.

Thus, the study of the operating modes of the ventilation equipment of the air conditioning system of transformed rooms of large volume is relevant.

To prepare the supply air to the room and maintain its parameters at a given level, a climate control system is used. It is obvious that for each purpose of the transformed room, the work of the engineering system should also be considered individually. Thus, the purpose of this study is to develop a single solution adapted to the multifunctional type of building.

In the room air conditioning system, equipment is used to prepare the supply air, which includes heat exchangers, elements of the cooling system, shut-off and control valves and other auxiliary elements. For large-volume public buildings, systems with the use of chillers are most often designed. The main role in the movement of heat and cold from the refrigerator to the treated air is performed by heat and cold carriers, the most popular is water. However, water as a coolant has a few disadvantages - it is a high crystallization temperature and large energy losses during transportation. When choosing a cooling medium for the study, preference is given to an aqueous solution of ethylene glycol, which has several advantages: there are different product concentrations, stable operating parameters and thermophysical properties over a long period, low crystallization temperature compared to water.

Based on the solutions presented in [1-2], a scheme of the supply air treatment system with an intermediate heat carrier [3] was developed (Fig. 1). A solution of ethylene glycol 40 is used as a heat and cold carrier \%. As follows from Fig. 1, the outdoor air with the parameters $t_{\text {out }}, \varphi_{\text {out }}, \mathrm{L}_{\text {out }}$ enters the heat exchanger of the recuperator of the supply system 1 , in which the process of heat exchange of air with an ethylene glycol solution takes place. After the recuperator 1 , the air enters the room with the parameters $t_{\text {in }}, \varphi_{\text {in }}, L_{\text {in }}$. Inside the unit, the ethylene glycol solution circulates through the small ring " heat exchanger of the refrigerating machine-recuperator $1 »$. The heat exchanger of the exhaust system 2 is switched off during the warm season with the help of a three-way tap 3 . The glycol solution is cooled in the heat exchanger of the refrigeration machine and uses the heat exchanger of the recuperator as an air cooler to cool the outside air. 


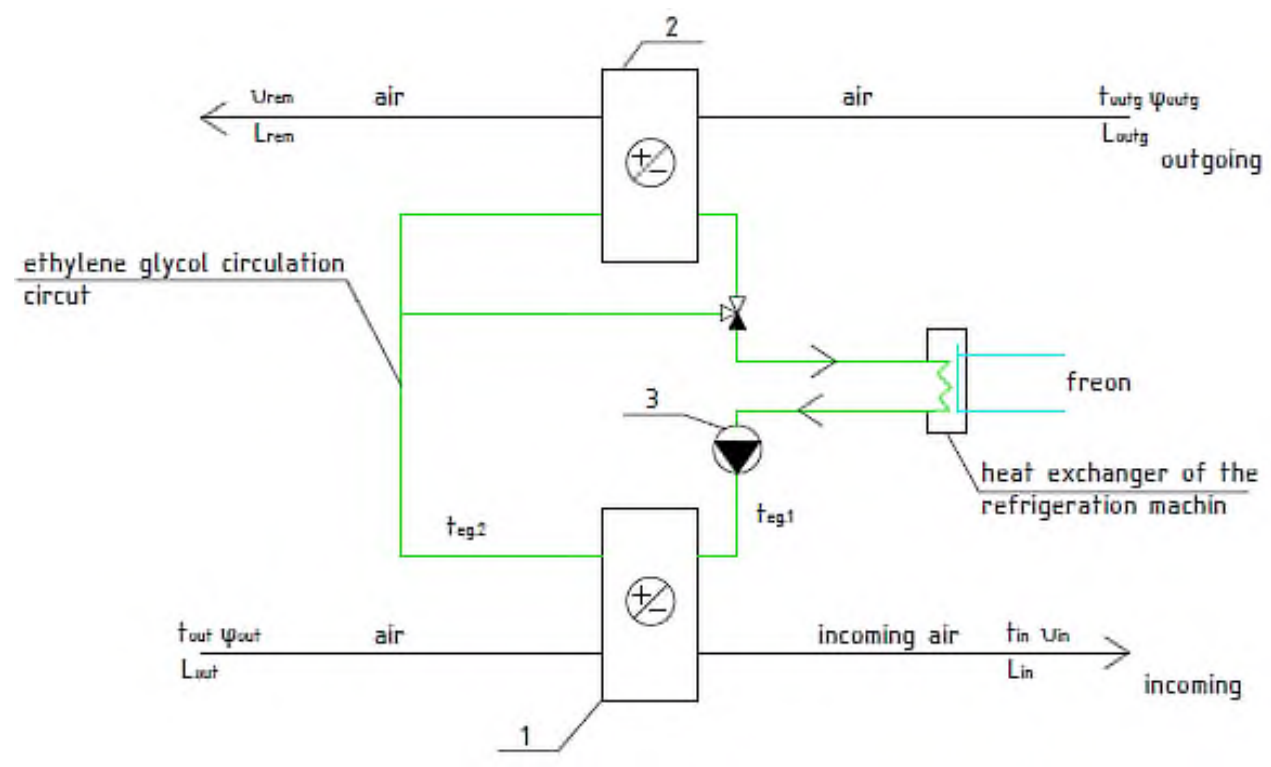

Fig. 1. Schematic diagram of the air treatment system with an intermediate heat and cold carrier in the warm season: 1 - supply system heat exchanger, 2 - exhaust system heat exchanger, 3 - three-way tap; tout, (out, Lout - temperature, ${ }^{\circ} \mathrm{C}$, relative humidity, $\%$, volume flow rate, $\mathrm{m}^{3} / \mathrm{h}$, the outgoing air; $t_{\text {rem, }} \varphi_{\text {rem }}, L_{\text {rem }}$ - temperature, ${ }^{\circ} \mathrm{C}$, relative humidity, $\%$, volume flow rate, $\mathrm{m}^{3} / \mathrm{h}$, the air to be removed; $t_{\text {in }}, \varphi_{\text {in }}, L_{\text {in }}$ - temperature, ${ }^{\circ} \mathrm{C}$, relative humidity, $\%$, volume flow rate, $\mathrm{m}^{3} / \mathrm{h}$, supply air; $t_{\text {out }}, \varphi_{\text {out }}, L_{\text {out }}-$ temperature, ${ }^{\circ} \mathrm{C}$, relative humidity, $\%$, volume flow rate, $\mathrm{m}^{3} / \mathrm{h}$, outdoor air; $t_{e g l}, t_{e g 2}-$ temperature of ethylene glycol at the inlet and outlet of the heat exchanger, accordingly, inside a closed loop.

\section{Materials and methods}

The object of the study is a multi-purpose concert hall «Event-Hall», located in Voronezh, with an area of 2100 square meters. As the initial data, the results presented in the [3]. HD state diagram of moist air builds processes of air treatment in Central air conditioning for all appointments of the room (Fig. 2), namely the red line - floor with full fit people (1), yellow line - dance orchestra from the podium (2), brown line - dance orchestra (3), green line - ring plus rostrum/ceremonial events (4), blue - exhibition hall (5). 


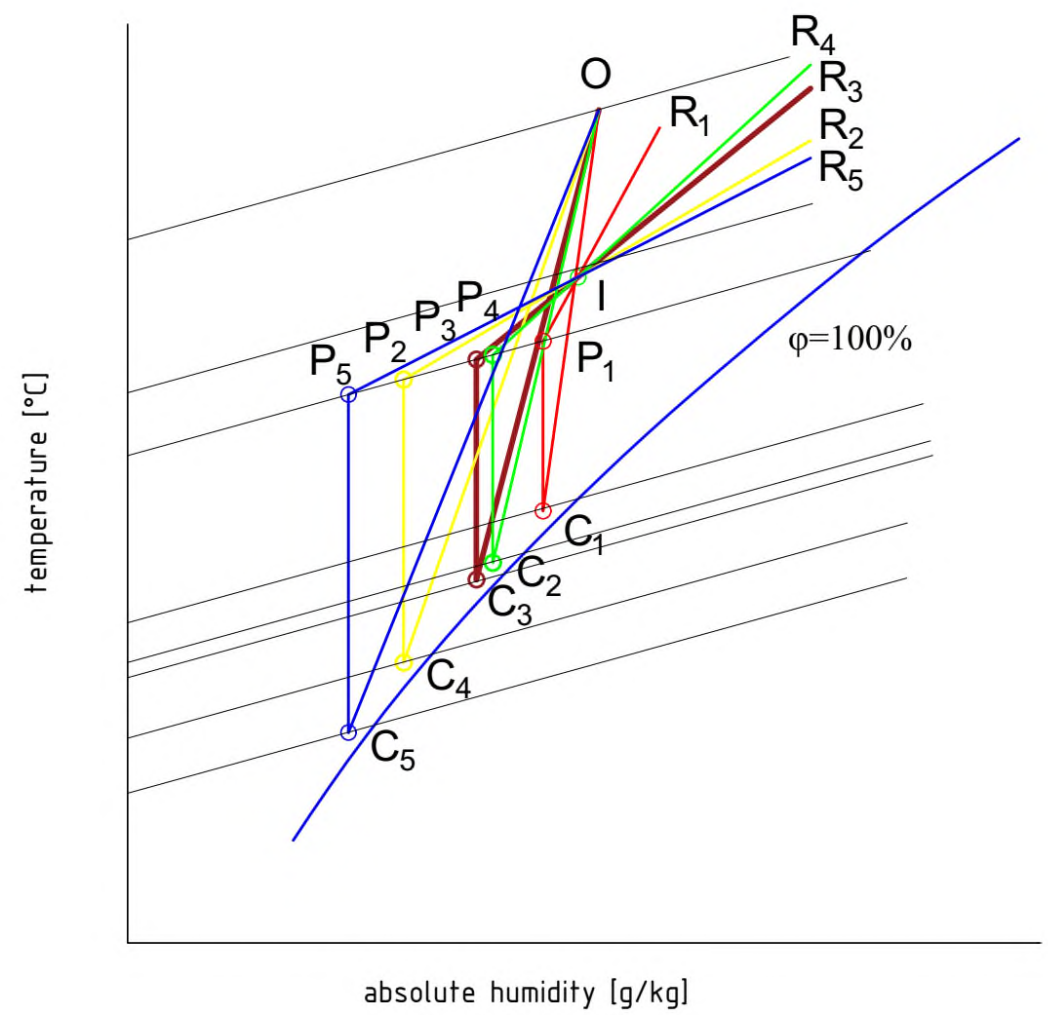

Fig. 2. Construction of air treatment processes in the central air conditioner in the warm season: dot $\mathrm{C}_{1}-\mathrm{C}_{5}$ - state of the air at the outlet of the heat exchanger, $\mathrm{P}_{1}-\mathrm{P}_{5}-$ the condition of the supply air, $\mathrm{R}_{1}$ $\mathrm{R}_{5}$ - condition of the outgoing air, $\mathrm{O}-$ the condition of the outside air

When preparing the supply air for a large volume transformed room intended for cultural events, it is necessary to carry out air treatment processes, namely, cooling and dehumidifying it in an air cooler.

The work of the heat exchanger - recuperator "air-ethylene glycol" when cooling the outside air for all operating modes of the object of study is analyzed.

To determine the maximum possible load on the elements of the building's air conditioning system, the type of transformed room with the highest supply air consumption was selected. The HD diagram shows the construction of the air treatment process at different outdoor temperatures (Fig. 3 and Fig. 4). 


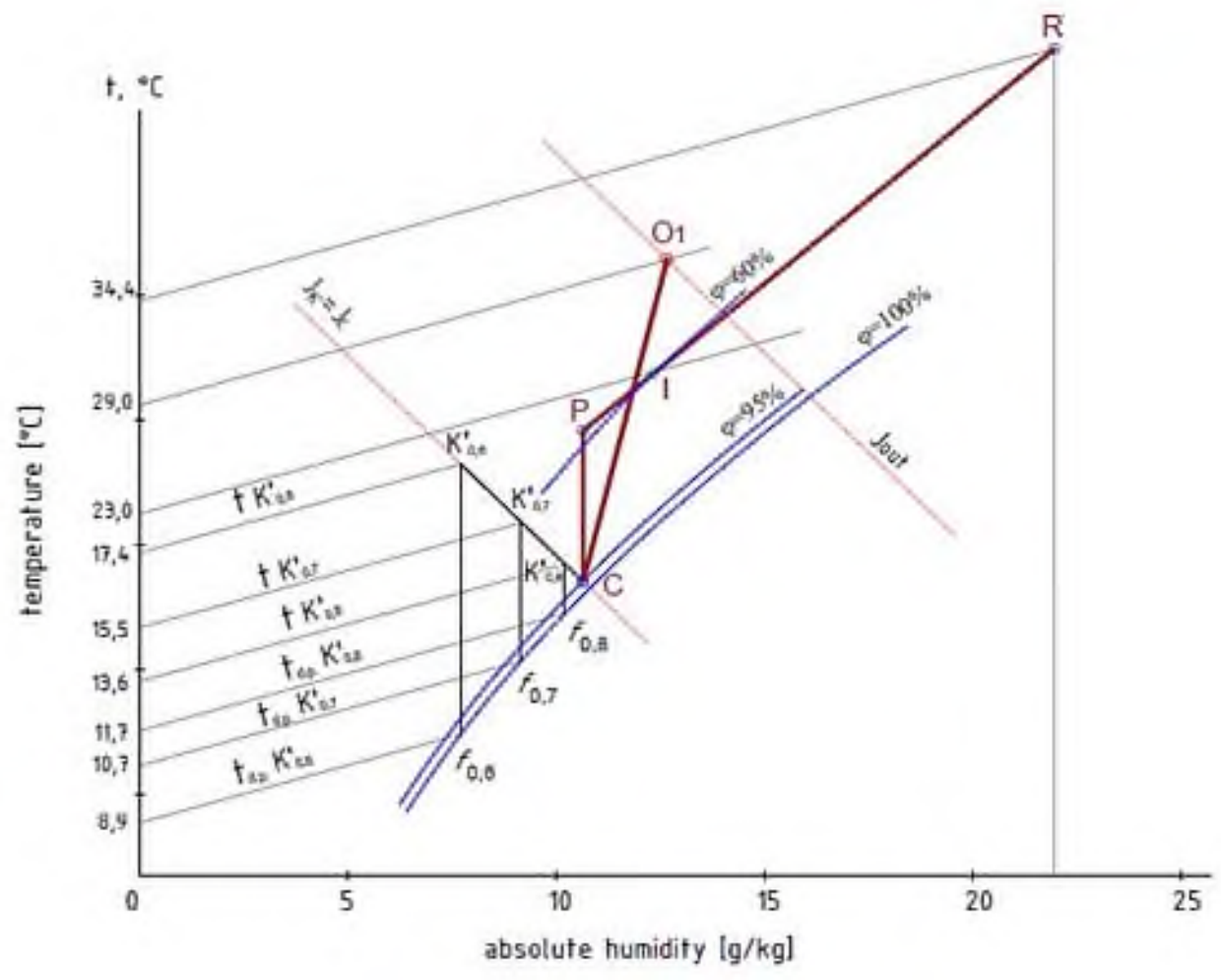

Fig. 3. Construction of air treatment processes in the central air conditioner in the warm season: $\operatorname{dot} C$ - condition of the air at the heat exchanger inlet, $\mathrm{P}$ - the condition of the supply air, $\mathrm{R}$ - condition of the outgoing air, $\mathrm{O}_{1}-$ the condition of the outdoor air at $t_{\text {out }}=29^{\circ} \mathrm{C}, t_{d . p}-$ dew point temperature, ${ }^{\circ} \mathrm{C}, f$ the state of the air at the dew point temperature, $t_{\mathrm{k}}$ ' - the initial air temperature in the "conventionally dry cooling», ${ }^{\circ} \mathrm{C}$. 


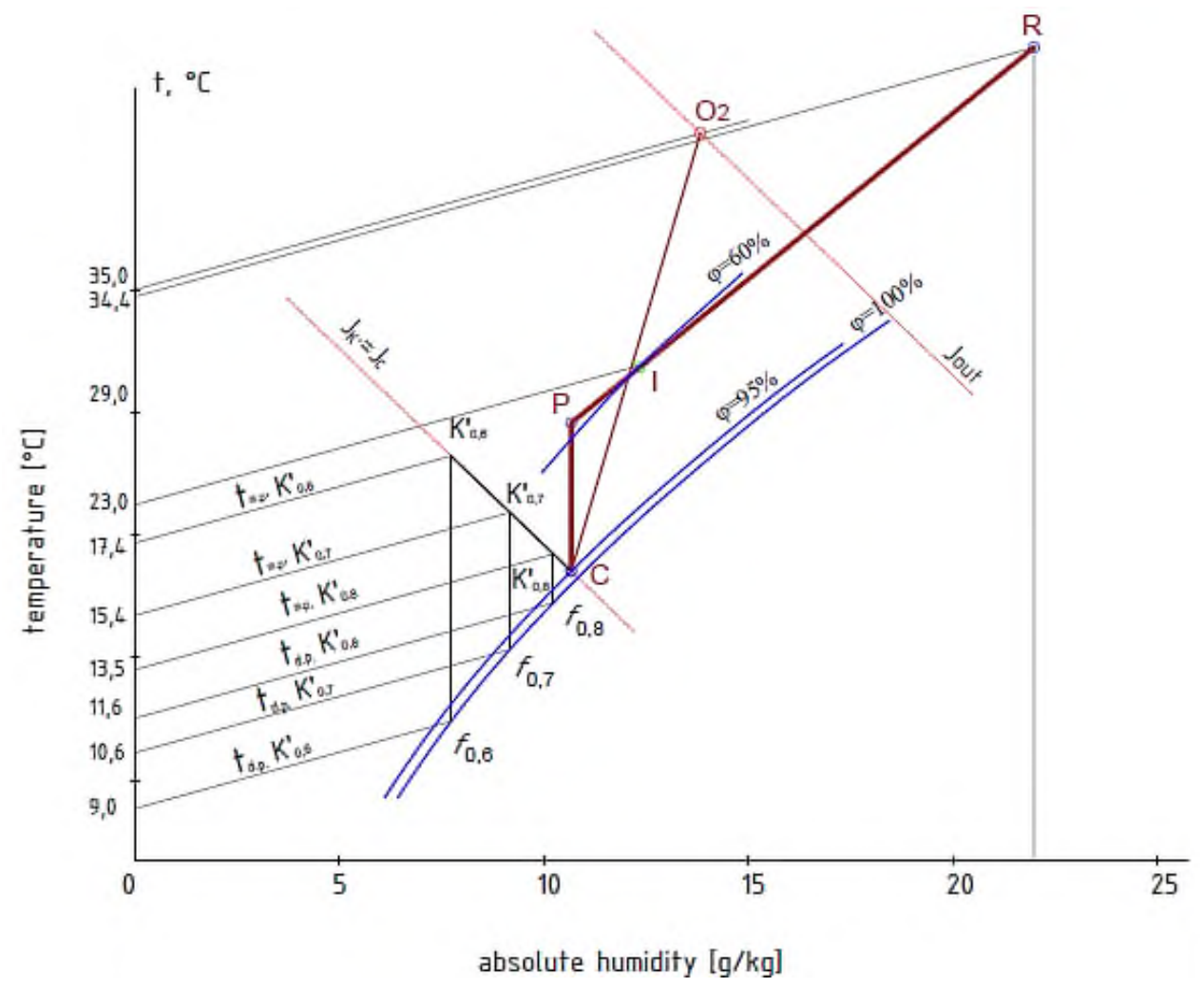

Fig. 4. Construction of air treatment processes in the central air conditioner in the warm season: dot $C$ - condition of the air at the heat exchanger inlet, $\mathrm{P}$ - the condition of the supply air, $\mathrm{R}$ - condition of the outgoing air, $\mathrm{O}_{2}-$ the condition of the outdoor air at $t_{\text {out }}=35^{\circ} \mathrm{C}, \mathrm{t}_{d . p}-$ dew point temperature, ${ }^{\circ} \mathrm{C}, \mathrm{f}-$ the state of the air at the dew point temperature, $t_{\mathrm{K}^{\prime}}$ - the initial air temperature in the "conventionally dry cooling», ${ }^{\circ} \mathrm{C}$.

Thus (Fig. 3, Fig. 4) the values of the temperature of the intermediate heat and cold carrier ethylene glycol, at the outlet of the heat exchanger are determined.

\section{Results}

The calculation method provides for the replacement of the real process of cooling and dehumidifying the air with a " conditionally dry cooling mode "[4], equivalent in terms of cold. It includes the determination of the parameters of the cooled air in the warm period of the year: initial $-G, t_{\mathrm{o}}, d_{\mathrm{o}}, J_{\mathrm{o}}, \varphi_{\mathrm{o}}$ final $-t_{\mathrm{c}}, d_{\mathrm{c}}, J_{\mathrm{c}}, \varphi_{\mathrm{c}}$.

In the HD diagram (Fig. 3, Fig. 4) the dew point temperature value is determined, which corresponds to the average temperature of the outer surface of the air cooler $t_{\mathrm{K}}$, ${ }^{\circ} \mathrm{C}$. The authors in Fig. 3, Fig. 4 show the construction of the real process of cooling and dehumidification of air and the " conditionally dry cooling mode». From the point K' (Fig. 3, Fig. 4) draw a line by $d_{K}=$ const before the intersection with $\varphi=95 \%$. As a result, a segment is obtained - corresponding to the beam of "conditionally dry cooling" of the air.

The actual parameters of the ethylene glycol temperature at the inlet and outlet of the heat exchanger are determined at different indicators of thermal efficiency according to the formula: 


$$
\Theta_{t}=\frac{t_{o}-t_{K^{\prime}}}{t_{o}-t_{\text {eg. } 1}}
$$

where $t_{\text {eg.1 }}$ - initial temperature of the medium at the outlet of the heat exchanger, ${ }^{\circ} \mathrm{C}$.

The temperature of ethylene glycol at the outlet of the heat exchanger is determined by the formula [5-8]:

$$
t_{K^{\prime}}=t_{o}-\Theta_{t} \cdot\left(t_{o}-t_{e g .1}\right)
$$

where $\Theta_{t}$ - the indicator of heat engineering efficiency, the values are accepted in the studies $0.6 ; 0.7 ; 0.8 ; 0.9$ and 1 .

We determine the final temperature of the medium at the outlet of the heat exchanger, ${ }^{\circ} \mathrm{C}$, using the formula:

$$
t_{e g .2}=t_{e g .1}+W\left(t_{o}-t_{c}\right)
$$

where $W$ - the index of the ratio of the heat capacity of the flows is determined by [9].

The results of the calculations $t_{k^{\prime}}, t_{e g .1}, t_{e g .2}$ for different outdoor air parameters, see Fig. 5 and Fig. 6.

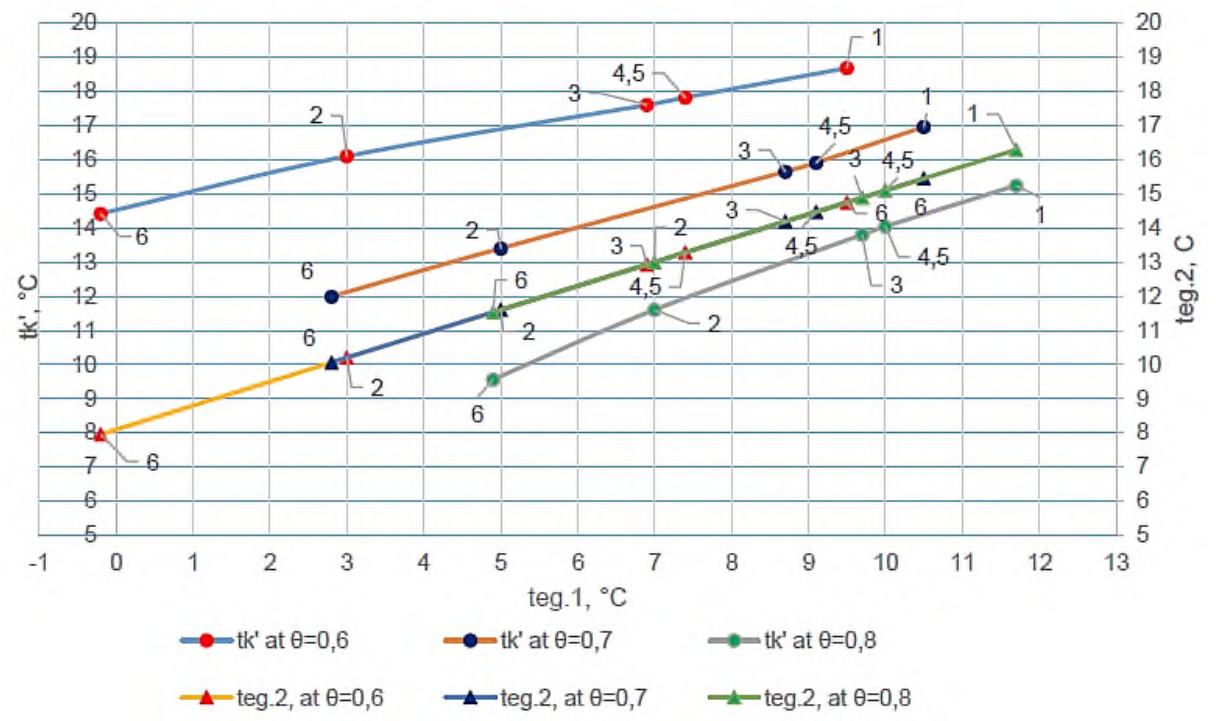

Fig. 5. Determination of the parameters of the intermediate heat and cold carrier depending on the heat and technical efficiency of the air treatment process at $t_{\mathrm{out}}=29^{\circ} \mathrm{C}$. 


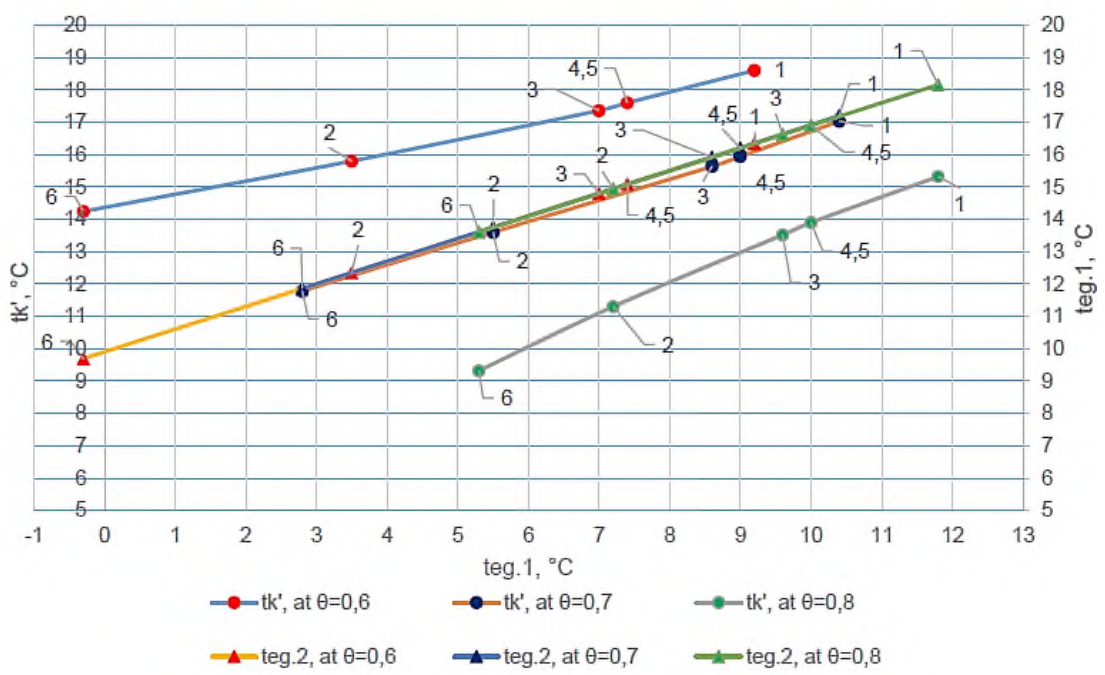

Fig. 6. Determination of the parameters of the intermediate heat and cold carrier depending on the heat and technical efficiency of the air treatment process at $t_{\text {out }}=35^{\circ} \mathrm{C}$

Thus, the dependence of the parameters of the heat exchange equipment and the operating modes of the transformed room is established. The regularities in the regulation of the system operation depending on the change in the load on the system are revealed.

The cooling capacity of the installation is determined depending on the modes of use of the room at the outdoor temperature according to the formula [9-11]:

$$
Q_{\text {refr }}=0.278 \cdot G \cdot\left(I_{o}-I_{c}\right),
$$

Where G- air consumption, $\mathrm{kg} / \mathrm{h} ; I_{o}$ the enthalpy of the outdoor air in the warm season, $\mathrm{kJ} / \mathrm{kg} ; I_{c}-$ enthalpy of air leaving the air cooler, $\mathrm{kJ} / \mathrm{kg}$.

The results of calculating the dependence of the ethylene glycol temperature at the inlet and outlet of the refrigerator on the cooling capacity $Q_{\text {refr }}$ are shown in Fig. 7 and fig. 8.

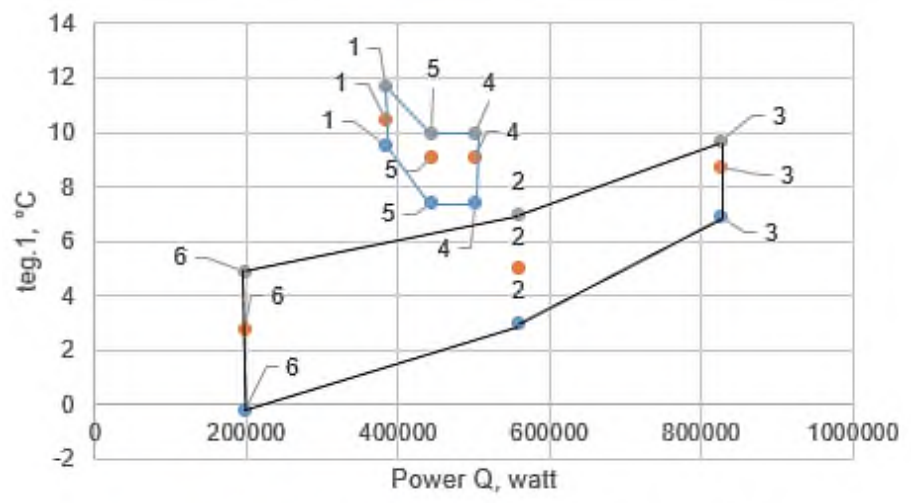

- teg. 1 , at $\theta=0,6$ eteg. 1 , at $\theta=0,7$ efeg. 1 , at $\theta=0,8$

Fig. 7. Calculated range of effective operation of heat exchange equipment at $t_{\text {out }}=29^{\circ} \mathrm{C}$ 


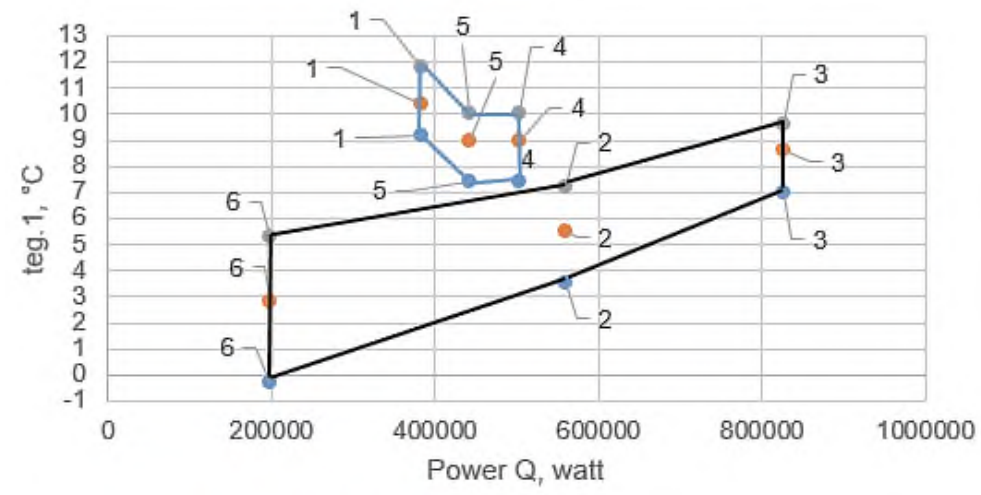

- teg. 1 , at $\theta=0,6 \bullet$ teg. 1 , at $\theta=0,7 \bullet$ teg. 1 , at $\theta=0,8$

Fig. 8. Calculated range of effective operation of heat exchange equipment at $t_{\mathrm{out}}=35^{\circ} \mathrm{C}$

Thus, the obtained results allow us to set the sequence of switching modes of heat exchange equipment depending on the parameters of the indoor environment in the desired range of thermal efficiency. As a result, it is possible to create an algorithm for the operation of the air conditioning system of the transformed room.

\section{Conclusions}

It is established that to ensure energy saving of the air conditioning system of multifunctional premises of public buildings, the most rational solution is the use of a cooling system with an intermediate heat carrier ethylene glycol.

Studies of the operating mode of heat exchange equipment in the permissible range of values of its thermal efficiency are carried out. The parameters of the intermediate heat and cold carrier - ethylene glycol-are determined, and their boundary values are established for the proposed installation for processing the supply air of the transformed room.

The operating ranges of the developed scheme for variable flow rate of the treated air and various parameters of the outdoor air are given. Based on the results obtained, it is possible to develop an algorithm for automating the ventilation system adapted to quickly transformable premises of cultural and entertainment buildings.

\section{References}

1. A. V. Sverdlov, AVOK: ventilation, heating, air conditioning, heat supply and construction thermophysics, 3, 28-30 (2014)

2. A. V. Sverdlov, AVOK: ventilation, heating, air conditioning, heat supply and construction thermophysics, 2, 22 (2015)

3. V. V. Shichkin, M. N. Zherlykina, K. V. Garmonov, S. A. Solovyov, Housing and communal infrastructure, 2(13), 46-56 (2020)

4. M. N. Zelikina, T. V. Shchukin, E. I. Lobov, Housing and utilities infrastructure, 3(6), 34-42 (2018)

5. J. Zemitis, A. Borodinecs, 4th International Conference on Innovative Materials, Structures and Technologies, 4, 12-19 (2019) 
6. A. A. Mershchiev, I. P. Mershchieva, Engineering systems and structures, 4, 16-21 (2013)

7. V. V. Shichkin, M. N. Zherlykina, S. A. Yaremenko, S. A. Soloviev, Housing and communal infrastructure, 1(12), 34-42 (2020)

8. D. V. Lobanov, V. V. Shichkin, Housing and communal infrastructure, 4(3), 70-75 (2017)

9. R. M. Lazzarin, A. Gasparella, APPLIED THERMAL ENGINEERING, 1-2, 47-67 (1998)

10. M. Zherlykina, E. Smorodina, G. Kalabukhov, A. Smolyaninov, E3S WEB OF CONFERENCES, 3, 11-17 (2020)

11. V. I. Livchak, AVOK: ventilation, heating, air conditioning, heat supply and building thermal physics, 6, 4-9 (2007) 